\title{
Misuse of benzodiazepines and Z-drugs in the UK
}

\author{
V. Kapil, J. L. Green, C. Le Lait, D. M. Wood and P. I. Dargan
}

\section{Summary}

Benzodiazepines and Z-drugs are commonly prescribed for insomnia and anxiety syndromes and there is increasing concern regarding their misuse. Using an internet-based questionnaire we found that of 1500 respondents $7.7 \%$ $(n=116)$ had misused one or more of these medications. Almost 15\% of those misusing at least one of these drugs did so once weekly or more often. The main reasons reported for their use were to help sleep (66.4\%), to cope with stress (37.1\%) and/or to get high (31.0\%). A total of 31\% obtained the medications from multiple sources; healthcare professionals (55.2\%) and friends/family (39.7\%) most commonly. Our study can be used to inform prevention measures for their misuse.

\section{Declaration of interest}

None.
Benzodiazepines are hypnotic drugs that enhance the activity of gamma-aminobutyric acid (GABA) at the $\mathrm{GABA}_{\mathrm{A}}$ receptor. In the UK, they are classified as Class $\mathrm{C}$ drugs under the Misuse of Drugs Act, 1971. Zolpidem, zopiclone and zaleplon are nonbenzodiazepine hypnotics that share a similar mode of action but are chemically distinct; they are more commonly known as Z-drugs. Currently in the UK, only zolpidem is controlled as Class C under the Misuse of Drugs Act, 1971 (although recently the UK Advisory Council for the Misuse of Drugs has recommended to the Home Secretary that Z-drugs should also be controlled). Both benzodiazepines and Z-drugs are cautiously recommended as shortterm treatments for insomnia and anxiety. ${ }^{1,2}$ Concerns regarding the addictive potential of these drugs have been highlighted for many years ${ }^{3,4}$ and the problems and sequelae of chronic, licit use of these drugs have been extensively reviewed elsewhere, ${ }^{5}$ including frequent implications in substance-misuse-related deaths in the UK. ${ }^{6}$ However, the prevalence of misuse of these drugs and the sources and/or reasons for their misuse in the general population is currently unknown. ${ }^{5}$ It is crucial to determine these issues in order that suitable interventions and public health campaigns can be formulated to tackle any problem identified effectively. In this study, we determined the epidemiology of misuse of these drugs through the use of an internet-based survey ${ }^{7}$ of the general UK population.

\section{Method}

The survey was delivered through a global market research company, GMI (www.gmi-mr.com/global-panel), which has an established global consumer panel of millions of persons worldwide. We devised a question-based survey that included questions about the medications of interest (included as an online supplement to this paper), from which an internet-based, browser-accessed questionnaire was created by the market research company. UKbased members of the established market research panels were invited by electronic means to complete our questionnaire; the survey was targeted at the age group 16-59 years. Answers were collected electronically until the study was capped after the collection of complete answers from a pre-determined 1500 individuals. At this point, the raw data were handed to us linked to a fully anonymised unique personal identifier. The data were formatted and collated into an electronic database and analysed. Basic demographics were collected together with data on lifetime prevalence and frequency of misuse of a range of benzodiazepines and Z-drugs. Where individuals had misused one of these drugs, they were asked to indicate the source(s) of supply for misuse, frequency of misuse and the reasons for misuse. We included similar questions (see online supplement) regarding the lifetime prevalence of use of classical recreational drugs (cocaine cannabis, 3,4-methylenedioxymethylamphetamine (MDMA, 'ecstasy')) so that we were able to benchmark our surveyed population against existing national data provided by the 2011/2012 Crime Survey for England and Wales. ${ }^{8}$

\section{Results}

The online questionnaire was completed by 1500 respondents, 737 males (49.1\%) and 763 females (50.9\%). The proportion of individuals in predetermined age ranges were: 9.1\% (16-20 years), $40.5 \%$ ( $21-39$ years), $21.1 \%$ ( $40-49$ years) and $29.3 \%$ (50-59 years).

Self-reported lifetime prevalence of use of MDMA was $8.2 \%$, cocaine $8.1 \%$ and cannabis $28.1 \%$. This compared with $8.6 \%$, 9.5\% and $31.0 \%$ respectively for these three drugs in the 2011/ 2012 Crime Survey for England and Wales. ${ }^{8}$

A total of $392(26.1 \%)$ of the 1500 respondents reported having ever taken a benzodiazepine or Z-drug. Of these, 116 $(29.6 \%)$ reported that they had ever misused a benzodiazepine of Z-drug (i.e. $7.7 \%$ of the total population). Thirty-four $(29.3 \%)$ of these 116 had taken a benzodiazepine or Z-drug under appropriate direction and also misused the same medicine at other times; $24(20.7 \%)$ had taken a benzodiazepine or Z-drug under medical direction as well as misusing at least one other benzodiazepine or Z-drug. Most individuals ( $n=69,59.5 \%)$ had only misused one specific benzodiazepine or Z-drug, whereas $47(40.5 \%)$ individuals had misused at least two of these medications (median 1; range 1-7). The mean number of benzodiazepines and Z-drugs misused was $1.6($ s.d. $=1.1)$.

Diazepam $(n=62,53.4 \%)$ and zopiclone $(n=28,24.1 \%)$ were the most frequently misused medications, with decreasing numbers of individuals misusing other medications, lorazepam $(n=26,22.4 \%)$, alprazolam $(n=20,17.2 \%)$, oxazepam $(n=14,12.1 \%)$, zaleplon $(n=13,11.2 \%)$, zaleplon $(n=13,11.2 \%)$, nitrazepam $(n=12$, $10.3 \%)$, phenazepam $(n=9,7.8 \%)$ and zolpidem $(n=6,5.2 \%)$.

Overall, most individuals misused benzodiazepines and Z-drugs less than once monthly $(n=65,56.0 \%)$. However, 34 (29.3\%) were misusing at least one benzodiazepine or Z-drug between once weekly to once monthly; 13 (11.2\%) individuals were misusing at least one benzodiazepine or Z-drug between once daily to once weekly; and $4(3.4 \%)$ individuals were misusing at least one benzodiazepine or Z-drug more than once daily. 
Respondents often gave multiple reasons for misuse of benzodiazepines and Z-drugs. The most frequently given reason for misuse was to help sleep $(n=77,66.4 \%)$. Similar proportions of respondents indicated that they took them to cope with stress $(n=43,37.1 \%)$, to get high $(n=36,31.0 \%)$ or for social reasons $(n=28,24.1 \%)$. Smaller numbers of respondents misused at least one benzodiazepine or Z-drug out of curiosity $(n=15,12.9 \%)$, to help come down from other drugs $(n=12,10.3 \%)$ or because they felt that taking these drugs was safer than taking street/illegal drugs $(n=12,10.3 \%)$

Most $(n=80,69.0 \%)$ respondents obtained the medication for misuse from a single source, but $36(31 \%)$ respondents obtained the medication for misuse from multiple sources (range 1-5). The most common source for benzodiazepines and Z-drugs for misuse was a prescription from a health professional $(n=64$, $55.2 \%)$. Other sources included: friends and/or family $(n=46$, $39.7 \%)$; bought from the internet $(n=31,26.7 \%)$; street dealers $(n=23,19.8 \%)$; and obtained from abroad $(13,11.2 \%)$.

\section{Discussion}

This study, to our knowledge, is the first UK study to estimate the prevalence of misuse of hypnotic medications. Our questionnaire cohort had a similar lifetime prevalence of use of classical recreational drugs to the overall UK population as surveyed through the Crime Survey for England and Wales. This suggests that our survey population is comparable with and representative of the general population in respect to their classical recreational drug use. Our results suggest that there is appreciable misuse of benzodiazepines and Z-drugs in the UK, with approximately $7.7 \%$ of respondents self-reporting misuse of any of these medications. This finding is consistent with similar results from data calculated from the National Comorbidity Study in the USA, which estimated life-time prevalence of misuse of sedative drugs of $7.1 \%$ among adults. ${ }^{9}$ In our survey, the majority of misuse appears to be from legitimately prescribed medication from health professionals, which has important implications for future strategies to reduce access to these medications. More awareness of this problem at physician and pharmacy levels, coupled with training and stratagems to uncover medication misuse (such as pill counting) are clearly urgently needed. ${ }^{10}$

Use of similar surveys such as these can be used to determine estimates of use or misuse of medications at a general population level and within population subgroups ${ }^{7}$ to help regulatory agencies appreciate the epidemiology of substance misuse. This would allow decisions regarding whether further control and/or monitoring on both the prescription and supply of the medications from health professionals and from other illicit sources, and also on the overall use of these medications, is required. In addition, further work is needed to understand the reasons for misuse of these medications, to enable appropriately targeted harm-minimisation activities through appropriate multi-agency responses.

V. Kapil, MA, MBBS, MRCP, PhD, Clinical Toxicology, Guy's and St Thomas' NHS
Foundation Trust and King's Health Partners, London, William Harvey Research
Institute, Centre for Clinical Pharmacology, Barts and the London School of Medicine
and Dentistry, Queen Mary University of London, London and Barts Hypertension
Clinic, Barts Health NHS Trust, London UK; J. L. Green, PhD, CCRP, C. Le Lait, MC,
MS, Denver Health Rocky Mountain Poison and Drug Center, Denver, Colorado, USA;
D. M. Wood, MB ChB(Hons), MD, FRCP, FACMT, FBPharmacols, P. I. Dargan, MBBS,
FRCP, FRCPE, FACMT, FAACT, FEAPCCT, Clinical Toxicology, Guy's and St Thomas'
NHS Foundation Trust and King's Health Partners, London and King's College London,
London, UK

Correspondence: Paul Dargan, Clinical Toxicology, 3rd Floor, Block C, South Wing Guy's and St Thomas' NHS Foundation Trust, St Thomas' Hospital, Westminster Bridge Road, London, SE1 7EH, UK. Email: Paul.Dargan@gstt.nhs.uk

\section{References}

1 National Institute for Health and Care Excellence. Guidance on the Use of Zaleplon, Zolpidem and Zopiclone for the Short-Term Management of Insomnia. Technology Appraisal 77. NICE, 2004 (http://www.nice.org.uk/ nicemedia/live/11530/32845/32845.pdf)

2 National Institute for Health and Care Excellence. Generalised Anxiety Disorder in Adults: Management in Primary, Secondary and Community Care. National Clinical Guideline 113. NICE, 2011 (www.nice.org.uk/nicemedia/live/ 13314/52667/52667.pdf)

3 Committee on Safety of Medicines. Benzodiazepines, dependence and withdrawal symptoms. Curr Probl 1988; 21: 1-4.

4 Jones IR, Sullivan G. Physical dependence on zopiclone: case reports. $B M J$ 1998; 316: 117

5 Lader M. Benzodiazepines revisited-will we ever learn? Addiction 2011; 106 : 2086-109.

6 Corkery J, Claridge H, Loi B, Goodair C, Schifano F. Drug-Related Deaths in the UK: January-December 2012. International Centre for Drug Policy, 2014

7 Kapil V, Green JL, Le Lait C, Wood DM, Dargan PI. Misuse of the GABA-Analogues Baclofen, Gabapentin and Pregabalin in the United Kingdom. Br J Clin Pharmacol 2013; 6 Nov ePub ahead of print (doi: 10.1111/bcp.12277).

8 Home Office Statistics Unit/Office for National Statistics. Drug Misuse Declared: Findings from the 2011/2012 Crime Survey for England and Wales. Home Office, 2013 (https://www.gov.uk/government/publications/drugmisuse-declared-findings-from-the-2011-to-2012-crime-survey-for-englandand-wales-csew-second-edition).

9 Goodwin RD, Hasin DS. Sedative use and misuse in the United States Addiction 2002; 97: 555-62.

10 National Center on Addiction and Substance Abuse at Columbia University. Under the Counter: The Diversion and Abuse of Controlled Prescription Drugs in the U.S. National Center on Addiction and Substance Abuse at Columbia University, 2005 (http://www.casacolumbia.org/addiction-research/reports/ under-the-counter-diversion-abuse-controlled-perscription-drugs). 